\title{
Estructuración del centro de gestión ambiental urbana desde un enfoque de sustentabilidad en el barrio san benito en bogotá, colombia. Primer paso estado del arte
}

\section{Environmental management center in the San Benito barrio from a focus of urban sustainability}

\author{
Efrén Danilo Ariza Ruiz ${ }^{1}$ \\ Corporación Universitaria Minuto de Dios UVD
}

\section{RESUMEN}

El presente artículo de revisión bibliográfica pretende construir un estado del arte que permita dotar de sustento teórico y metodológico una actuación local en el Barrio San Benito de Bogotá en la localidad de Tunjuelito, protegiendo los recursos hídricos, regulando de manera coherente el uso del suelo, reduciendo la contaminación en el aire, a través de la estructuración y puesta en marcha del CENTRO DE GESTIÓN AMBIENTAL URBANA EN EL

1 Economista Universidad Nacional de Colombia, Egresado Maestría en Sociología Universidad Nacional de Colombia, Magister en Filosofía Latinoamericana Universidad Santo tomas, PH. D ๔ en Filosofía Universidad Santo Tomas. Docente investigador Grupo RADCO Líder Semillero de Investigación Contabilidad y Consecuencias Ambientales de la Cultura Corporación Universitaria Minuto de Dios UVD. efren.ariza@uniminuto.edu.co https://orcid.org/0000-0001-7762-3562
BARRIO SAN BENITO DESDE UN ENFOQUE DE SUSTENTABILIDAD URBANA.

PALABRAS CLAVE: Sustentabilidad, Gestión Ambiental Urbana, Sustentabilidad Urbana, Responsabilidad Social Empresarial, Políticas Públicas.

CLASIFICACIÓN JEL: Q57, Q58, Q26, Q28, Q38.

\section{ABSTRACT}

This article of bibliographic review aims to build a state of the art, which allows to provide theoretical and methodological sustenance a local performance in the Barrio San Benito de Bogotá in the town of Tunjuelito, protecting water resources, consistently regulating land use, reducing pollution in the air, through the 
structuring and implementation of the URBAN.

\section{KEYWORDS: Sustainability, Urban} Environmental Management, Urban Sustainability, Corporate social responsibility, Public policies.

JEL CLASIFICATION: Q57, Q58, Q26, Q28, Q38.

\section{INTRODUCCIÓN}

La construcción del presente Estado del arte busca responder a la pregunta ¿Cómo estructurar un Centro de Gestión Ambiental Urbana en el barrio San Benito, desde un enfoque de sustentabilidad que aporte al fortalecimiento de una política pública ambiental?

En la segunda mitad del siglo $\mathrm{XX}$, Colombia vivió una fuerte tendencia a la urbanización. Mientras en 1950 la población urbana correspondía al $39 \%$ del total de la población, en el 2005 ascendió al $76 \%$ y se estima que en el 2020 superará el $80 \%$. Estos asentamientos se localizan, en su mayoría, a lo largo de tres ejes urbanos en las regiones andina, atlántica y sur occidente, con una progresiva conurbación y metropolización alrededor de las grandes ciudades (cifras incluidas en el Plan Nacional de Desarrollo, 2007). "Durante los próximos 15 años, las ciudades colombianas tendrán una fuerte presión demográfica. Para 2019, se estima que la población en cabeceras se incrementará en 10 millones de habitantes, el número de ciudades con más de 1 millón de habitantes pasará de 4 a 7 y las de más de cien mil habitantes pasarán de 37 a 55 , lo que consolidará aún más el sistema de ciudades de nuestro país (Ministerio de Ambiente, Vivienda y Desarrollo Territorial, 2008, Pág. 7)

Un área de investigación con especial crecimiento en las ciencias económicas es la economía urbano regional, campo que ha consolidado el concepto economías de aglomeración. Como consumidor, un habitante de Bogotá identifica a-priori a la realización de su compra, los lugares a los cuales puede dirigirse para adquirir el bien o servicio que busca. Por ejemplo, si busca muebles se dirige a el 12 de octubre, la autopista norte o Venecia, o si busca rumba y entretenimiento se dirige a la calle 81, el parque de la 93 o "Cuadra picha", este concepto explica la dinámica del Barrio San Benito. "El barrio San Benito se encuentra al sur de la ciudad de Bogotá, pertenece a la localidad de Tunjuelito fue fundado en 1948, por curtidores de Chocontá y Villa Pinzón que en busca de agua de asentaron alrededor del río Tunjuelito" (Romero Manrique \& Leguizamón Cruz, 2016, pág. 34).

En el suroccidente de Bogotá entre los barrios San Benito y el Tunal principalmente se calcula que funcionan cerca de 400 microempresas que trabajan el cuero de una manera industrial, labor que se desarrolla en el sector desde los años 30 , si bien es este un próspero sector industrial de la ciudad y del país, es también un foco del daño ambiental y social generado desde la industria, uno de los principales daños ambientales se genera por el vertimiento de cromo y otros desechos químicos al río Tunjuelito. (Zorro Sánchez, 2020, pág. 107)

Este impacto ambiental genera un claro impacto social en el aspecto de la salud especialmente de los menores de edad, que presentan enfermedades respiratorias y epidemias y brotes cutáneos; dicha situación se presenta en los barrios más cercanos al río, así como la evidencia de un volumen significativo de residuos sólidos y orgánicos generados por la industria, esto no solo afecta la apariencia del sector, sino la 
permanencia de animales de carroña y jaurías de perros atraídos por los olores generados, otra afectación social es la valorización comercial de los predios pues cuando algunos vecinos del sector han querido vender dichos predios han debido enajenarlos por montos menores a su avalúo catastral. (Ramírez, J. A. 2014).

Los elementos anteriores evidencian lanecesidad de aportar en la construcción de una política pública ambiental urbana que como señala Duquino-Rojas (2018) debe fundamentarse en la complejidad de la naturaleza y de sus procesos internos de producción (de biomasa y de carácter neguentrópico). Tal complejidad desborda la instrumentalidad a la que ha sido reducida desde la tecnocracia y el modelo económico, y plantea la exigencia de aparatos de aprehensión más integrales, capaces de dar cuenta de la amplia red de relaciones que se entreteje en los diversos fenómenos ambientales que tienen lugar en los territorios.

En este sentido, el presente artículo de revisión presenta unos antecedentes, un marco teórico donde se evidencian lo que se ha escrito sobre Gestión ambiental urbana desde un enfoque de sustentabilidad, una discusión sobre el método y unos resultados donde se evidencian los elementos de los que constaría nuestra propuesta de CENTRO DE GESTIÓN AMBIENTAL URBANA EN EL BARRIO SAN BENITO DESDE UN ENFOQUE DE SUSTENTABILIDAD.

\section{ANTECEDENTES Y REVISIÓN DE LITERATURA}

La gestión ambiental urbana, se puede definir como "un conjunto de acciones encaminadas a lograr la máxima racionalidad en el proceso de decisión relativo a la conservación, defensa, protección y mejora del medio ambiente, a partir de un enfoque interdisciplinario y global" (Ministerio de Ambiente, Vivienda y Desarrollo Territorial, 2008, Pág. 17)
Reconociendo que es un avance el diseño de una política de gestión ambiental urbana, es necesario reconocer que dicha política se realiza desde un enfoque de sostenibilidad débil (desarrollo sostenible) que busca enfrentar la crisis ambiental desde las coordenadas de la globalización neoliberal y una sostenibilidad fuerte que aboga por la búsqueda de tipos de organización económica alternativos al capitalismo, como lo señalan Ariza Buenaventura (2007); Ariza Ruiz (2019); Gorz (2008); Narciso (2018); García \& Vergara (2000) ; Carter, (1996)

Se establece que la sostenibilidad débil conserva una relación de manera proporcional con el medio ambiente, ya que a mayor crecimiento existe una mayor sensibilidad ambiental, debido a los avances tecnológicos y la optimización de recursos determinando que el desarrollo económico es establecido como una meta para lograr el crecimiento sostenible.

En la siguiente tabla Carter, expresa la transición que ha tenido la sostenibilidad en los entornos políticos, económicos, sociales y de discurso. 
Tabla 1. Sostenibilidad débil y fuerte

\begin{tabular}{|c|c|c|c|c|}
\hline $\begin{array}{l}\quad \text { Fase } \\
\text { 1. } \\
\text { Sostenibilidad } \\
\text { muy Débil }\end{array}$ & $\begin{array}{l}\quad \text { Políticas } \\
\text { Palabras } \\
\text { no sinceras } \\
\text { respecto a la } \\
\text { integración de } \\
\text { las políticas. }\end{array}$ & \begin{tabular}{|l|}
\multicolumn{1}{|c}{ Economía } \\
Retoques \\
menores con \\
instrumentos \\
económicos.
\end{tabular} & \begin{tabular}{|l|}
\multicolumn{1}{|c}{ Sociedad } \\
Conciencia \\
difusa y poca \\
cobertura en \\
los medios de \\
comunicación.
\end{tabular} & $\begin{array}{l}\text { Discurso } \\
\text { Grupos de } \\
\text { discusión } \\
\text { corporativas; } \\
\text { ejercicios de } \\
\text { consultas. }\end{array}$ \\
\hline $\begin{array}{l}2 . \\
\text { Sostenibilidad } \\
\text { Débil }\end{array}$ & $\begin{array}{l}\text { Integración } \\
\text { formal de } \\
\text { políticas y } \\
\text { objetivos } \\
\text { factibles. }\end{array}$ & $\begin{array}{l}\text { Reestructuración } \\
\text { sustancial de } \\
\text { los incentivos } \\
\text { microeconómicos. }\end{array}$ & \begin{tabular}{|l} 
Mayor \\
educación \\
pública con \\
visión de \\
futuro.
\end{tabular} & $\begin{array}{l}\text { Mesas redondas; } \\
\text { grupos de } \\
\text { representantes } \\
\text { (stakeholders); } \\
\text { control } \\
\text { parlamentario. }\end{array}$ \\
\hline $\begin{array}{l}3 . \\
\text { Sostenibilidad } \\
\text { Fuerte }\end{array}$ & $\begin{array}{l}\text { Obligando a la } \\
\text { integración de } \\
\text { las políticas y } \\
\text { la consecución } \\
\text { de acuerdos } \\
\text { internacionales. }\end{array}$ & $\begin{array}{l}\text { Evaluación } \\
\text { completa del } \\
\text { coste de la vida } \\
\text { en el planeta: } \\
\text { contabilización } \\
\text { "verde" en la } \\
\text { contabilidad } \\
\text { nacional. }\end{array}$ & \begin{tabular}{|l|} 
Integración \\
curricular; \\
iniciativas \\
locales como \\
parte del \\
crecimiento de \\
la comunidad.
\end{tabular} & $\begin{array}{l}\text { Implicación de } \\
\text { la comunidad; } \\
\text { hermanamiento } \\
\text { de iniciativas en el } \\
\text { mundo desarrollado } \\
\text { y en desarrollo. }\end{array}$ \\
\hline $\begin{array}{l}4 . \\
\text { Sostenibilidad } \\
\text { Muy Fuerte }\end{array}$ & $\begin{array}{l}\text { Encadenar } \\
\text { los convenios } \\
\text { internacionales; } \\
\text { obligaciones } \\
\text { nacionales de } \\
\text { cuidado; apoyo } \\
\text { legal cultural. }\end{array}$ & $\begin{array}{l}\text { Cambio formal a } \\
\text { una contabilidad } \\
\text { económica } \\
\text { sostenible } \\
\text { nacional e } \\
\text { internacional. }\end{array}$ & \begin{tabular}{|l|} 
Cambio \\
cultural amplio \\
acoplado con \\
la innovación \\
tecnológica \\
y las nuevas \\
estructuras \\
comunitarias.
\end{tabular} & $\begin{array}{l}\text { Las iniciativas } \\
\text { dirigidas } \\
\text { comunitariamente, } \\
\text { llegan a ser la } \\
\text { norma. }\end{array}$ \\
\hline
\end{tabular}

Fuente: Tomado de Carter, 1996. 
Las críticas al concepto de desarrollo sostenible, o sostenibilidad débil ha permitido construir una categoría llamada sostenibilidad fuerte, o enfoque de sustentabilidad Naredo (1994); Leff (2009); Carter (1996).

Tabla 2. Diferencias sostenibilidad débil y sostenibilidad fuerte

\section{SOSTENIBILIDAD DÉBIL}

- Concepto más antropocéntrico (tecnocéntrico) que ecocéntrico.

- Concepto mecanicista.

- Sostenibilidad como sinónimo de viabilidad del sistema socioeconómico.

- Sostenibilidad compatible con crecimiento económico.

- Capital natural sustituible por capital humano. Constancia del capital total.

- La sustitubilidad exige monetizar el medio natural.

- Creencia en un desarrollo sostenible, que en realidad es sostenido.

- Medioambiente local.

\section{SOSTENIBILIDAD FUERTE}

- Concepto más ecocéntrico que antropocéntrico.

- Concepto sistémico.

Sostenibilidad como relación variable entre el sistema socioeconómico y el ecosistema.

- Sostenibilidad incompatible con crecimiento.

- Capital natural complementario del no sustituible por capital humano. Constancia del capital natural.

- Muchos recursos, procesos y servicios naturales son monetariamente inconmensurables.

- Diversas evoluciones sostenibles (históricamente han existido).

- Medioambiente global y sistémico.

Fuente: Carter (1996)

El discurso de la Sustentabilidad, que nace desde la economía ecológica y la ecología política, pero hoy día es transversal a todas las ciencias, se convierte en un eje central para orientar iniciativas que contribuyan a un mejor equilibrio entre el hombre y la naturaleza y a la coexistencia entre nosotros mismos y las demás especies. En este sentido:

Una mirada atenta a los grupos humanos que impulsan cada tipo de desarrollo revela que mientras el desarrollo sostenible es liderado desde arriba por gobernantes de derecha, empresarios y asesores o consultores a su servicio, el desarrollo sustentable se promueve desde abajo, con las bases, desde grandes masas agobiadas por el sistema imperante $\mathrm{y}$, por tanto, es la sociedad civil con los campesinos, indígenas, trabajadores, afrodescendientes, desplazados $\mathrm{y}$ en general las clases populares marginadas quienes han comenzado a levantar las banderas de la sustentabilidad. (Valenzuela Jiménez, 2017, pág. 216)

Una nueva política pública ambiental debe estar basada en el derecho inalienable a 
la ciudad para todos los integrantes de la sociedad y en la reivindicación de unos valores humanos que trasciendan las intenciones políticas, la normatividad y la planeación urbana para convertirse en herramientas reales de empoderamiento social para la gestión y la administración de la ciudad. (Duquino-Rojas, 2018, pág.149).

\section{MÉTODO}

Un Estado del arte es una investigación documental en sí misma, una investigación documental, que busca identificar con precisión lo que han dicho otros autores sobre la temática a la que pertenece su investigación, es decir, estructuración de Centros de Gestión Ambiental urbanos desde un enfoque de sustentabilidad. Lo anterior permite abordar nuestra propuesta desde ángulos inéditos que incrementan el conocimiento en nuestro campo de formación, lo que otorga pertinencia a nuestra investigación. La revisión sistemática se realiza en bases de datos académicas como Google scholar, proquest, Dialnet, science direct y scopus con las palabras clave gestión ambiental urbana, sustentabilidad, y servicios ecosistémicos ambientales, tomando como criterios de inclusión aportes teóricos o metodológicos en términos de construcción de indicadores; se construyen fichas bibliográficas, fichas de trabajo y se sistematiza la información a través de RAES.

\section{RESULTADOS}

Los resultados de la revisión sistemática de literatura se dividen en tres dimensiones, la primera en lo referente al contexto de ejecución de la propuesta de Centro de Gestión Ambiental Urbana con enfoque de sostenibilidad, una segunda dimensión hace referencia a los elementos conceptuales y de política pública ambiental que soportan la mencionada propuesta; la última dimensión, hace referencia a los indicadores que permitan evaluar la gestión del centro propuesto y la evolución de las variables ambientales en el barrio San Benito a partir de su puesta en marcha.

\section{CONTEXTO EJECUCIÓN DE LA PROPUESTA}

Diversos estudios dan cuenta de las problemáticas ambientales que genera la actividad humana en el Barrio San Benito especialmente las relacionadas con las empresas transformadoras del cuero violando abiertamente la ley en cuanto al manejo de sus residuos sólidos y la contaminación del agua en cuanto al uso de elementos químicos que dañan el agua Montes Medina (2018); Vázquez y González (2009).

Hernandez Vargas \& Moreno Sierra (2017) en calidad de pasantes participan en el Proyecto Ambiental "Súmate al Cambio: Tunjuelito Por un Ambiente más sano"; que se estructuró en cuatro (4) componentes que abarcan políticas ambientales contempladas en el Plan integral de Gestión Ambiental PIGA y adoptadas por la Secretaria Distrital de Integración Social SDIS para ser implementado en la Subdirección Local de Tunjuelito. Los lineamientos Ambientales son: cero desperdicios de papel, cero desperdicios de agua, cero desperdicios de energía y cero residuos. Como segundo componente, se tiene el manejo adecuado de los residuos inorgánicos. El tercer componente es un proceso de compostaje para el manejo adecuado de los residuos orgánicos. El último componente hace referencia a la recuperación y embellecimiento de espacios, en especial en los jardines infantiles, para generar conciencia a la primera infancia y mitigar gases de efecto invernadero, se cumple instalando muros verdes, jardines verticales y cercas vivas.

Desde el programa de Contaduría Pública modalidad virtual y distancia UVD, específicamente desde el semillero de investigación "Contabilidad y Consecuencias Ambientales de la Cultura" se han adelantado 
dos investigaciones específicas en el barrio San Benito, la primera adelantada por Montes Medina (2018) en la que se diseña un tablero de mando Balance Scorecard ambiental aplicado a una curtiembre del Barrio y la segunda adelantada por Zorro Sánchez. (2020) donde se identifica la posicón contable de 96 empresas del curo ubicadas en el barrio,frente a la contaminación que generan con su actividad.

Zorro Sánchez (2020) identifica que los empresarios y dirigentes de las curtiembres ubicadas en el barrio San Benito de Bogotá, no muestran decididas posiciones de cambio hacia el respeto de la normatividad nacional e internacional de respeto al medio ambiente, generando un importante daño ambiental. El principal impacto se dá sobre los recursos hídricos al verter sustancias como cromo, alumbre, aluminio y otras sustancias químicas al Rio Tunjuelo, así mismo se genera un impacto social en el aspecto de la salud especialmente de los menores de edad, que presentan enfermedades respiratorias y epidemias y brotes cutáneos, en los barrios más cercanos al río, así mismo se evidencia la emisión de un volumen significativo de residuos sólidos y orgánicos generados por la industria, esto no solo afecta la apariencia del sector, sino que implica la permanencia de animales de carroña y jaurías de perros atraídos por los olores generados.

El trabajo de campo, realizado desde el Semillero de Investigación Contabilidad y Consecuencias Ambientales de la Cultura del Programa de Contaduría UVD de la Corporación Universitaria Minuto de Dios, es el que nos anima proponer la creación de un Centro de Gestión ambiental desde un enfoque de sustentabilidad en el Barrio San Benito

\section{CONCEPCIÓN E IMPLICACIONES DE POLÍTICA}

Desde el Ministerio de Ambiente Vivienda y Desarrollo Territorial (2008) se reconoce que
$\mathrm{Si}$ bien las áreas urbanas son vistas como expresión de oportunidades de desarrollo económico y social, también es cierto que la conformación del sistema urbano colombiano se ha dado con una escasa planificación ambiental o de consideraciones ambientales, lo que ha derivado en innegables costos para el medio ambiente, tanto por los desordenados procesos de ocupación, como porlas fuertes demandas de recursos que conllevan. Por otro lado, el desarrollo de múltiples actividades económicas y de servicios en el territorio urbano tienden a acumular los problemas ambientales inherentes al desarrollo urbano, al punto que la problemática ambiental de los grandes centros urbanos se ha convertido en factor determinante del deterioro de la calidad del hábitat urbano, la salud y el bienestar de sus habitantes, con otras posibles consecuencias indirectas sobre el medio ambiente nacional y global. (pág $5)$.

Duquino-Rojas (2018) realiza una crítica a la postura adoptada por la institucionalidad, denuncia la subyugación irrestricta a la retórica del desarrollo sostenible impuesta desde los espacios de poder transnacional, que hace que Colombia y Bogotá lleven 25 años sujetas a una política pública que, a la luz de los acontecimientos, ha fracasado, y no ha logrado mediar las disputas territoriales y económicas entre los intereses privados y públicos por los recursos naturales, y los valores ambientales (pág. 143). En este sentido se constituye en un referente en términos del enfoque y las variables a tener en cuenta por nuestra propuesta.

Así mismo en consonancia con Narciso (2018) nuestra propuesta de Centro de Gestión Ambiental urbana desde un enfoque de sustentabilidad, reconoce que el urbanismo y 
la planeación urbana no han considerado las relaciones socio-territoriales,

Por eso las propuestas de intervención en las ciudades han sido desastrosas, y no se debe necesariamente a la falta de instrumentos de planeación, sino más bien a las contradicciones que los mismos presentan, la falta de continuidad y seguimiento entre la representación de la realidad y los lineamientos de los planes. Asimismo, subyace una "aplicación" que resguarda el beneficio del poder económico, o sea, una visión de planeamiento y gestión fragmentada, descontextualizada y elitista, que ha sido manejada mediante una visión instrumentalista y una centralización de las decisiones y de los recursos. (pág. 142)

Es desde esta reflexión que Narciso (2018) enuncia un concepto fundamental la Estructura Ecológica Urbana:

Es un instrumento de gestión y planeación territorial fundamental parala regeneración de los sistemas naturales y artificiales del paisaje urbano y además sobresale por su carácter integral y procesual. Sin embargo, es relevante aclarar que la EEU no pretende ser un plano de intenciones o un modelo estático de planeación, sino más bien una estrategia de desarrollo urbano, cualificado, verde y urbano, que se teje a través de una red de sistemas de espacios verdes públicos, para asegurar la diversidad biológica de los ecosistemas que aún persisten en la ciudad, la fluidez controlada del drenaje hídrico y atmosférico, así como la densidad adecuada de las áreas edificadas (Telles, 1997). Asimismo cabe resaltar, al igual que Lezama y Domínguez (2006), "que no existe un tipo ideal de ciudad sostenible, sino que ésta se conforma de acuerdo con sus características ambientales y regionales, a la par que con sus condiciones sociales y económicas, reconociendo que no todas las ciudades comparten las mismas problemáticas". Como nos dice Massey y Allen (1984), "la geografía importa porque cada lugar es un lugar con características propias que lo definen e identifican".(pág.146)

Duquino-Rojas (2018) señala que una política ambiental territorial debe partir de la posibilidad de hacer una lectura más profunda de la realidad ambiental de un contexto geográfico, en sus continuidades y particularidades, de las potencialidades de este y de los diversos saberes, incluyendo en condiciones de alteridad los saberes ancestrales que reposan en él, como formas históricas más sustentables de ocupar y producir en el territorio, distantes de los procesos altamente entrópicos del desarrollo económico a ultranza.

Debe incluir la posibilidad de mirar la problemática ambiental desde la complejidad de su propia naturaleza, de la interacción de todas las dimensiones que componen y entretejen el territorio, para que tenga una coherencia estructural que pueda atender el impacto de las externalidades negativas sobre el medio ambiente, fruto de los procesos de explotación económica y del orden social instituido. Duquino-Rojas, 2018, pág.148)

Una nueva política pública ambiental debe estar basada en el derecho inalienable a la ciudad para todos los integrantes de la sociedad y en la reivindicación de unos valores humanos que trasciendan las intenciones políticas, la normatividad y la planeación urbana para convertirse en herramientas reales de empoderamiento social para la gestión y la administración de la ciudad. (Duquino-Rojas, 2018, pág.149) 
Esto es precisamente en lo que consideramos que podemos aportar con lo que buscamos con la creación del Centro de Gestión urbana desde un enfoque de sustentabilidad en el Barrio san Benito.

Hernández Garzón (2019) realiza un análisis territorial de la problematica ambiental urbana en el municipio de Facatativa, cercano a Bogotá, que hace parte de la región Sabana de Occidente y señala que

La forma como han actuado las diversas fuerzaseconómicas, sociales, políticas e ideológicas, tanto nacionales como internacionales, en el marco de laconsolidación y la ampliación del desarrollo capitalista en el país, han incidido en esta nueva conformación espacial que ha traído consigo una serie de complejas formas de territorialización, expresadas en la urbanización y la industrialización de la sabana, que son determinantes en los cambios en el estilo de vida, los imaginarios y las prácticas culturales, las estructuras productivas y el medioambiente de la región.(pág. 95)

Así mismo Hernández Garzón (2019), señala un aspecto importante para abordar la gestión ambiental urbana. "El enfoque conceptual que sustenta la comprensión de los problemas ambientales urbanos exige una visión holística del territorio que hace indispensable la integración de la geohistoria, la geopolítica y la dimensión ambiental" (pág. 96)

El análisis de los problemas ambientales desde el punto de vista geopolítico permite conocer los fenómenos de índole económica, social, política y cultural que se producen y a la vez se reproducen el territorio, con el fin de determinar las posibles interrelaciones entre estos y sus efectos. El abordaje de los problemas ambientales debe considerar las fuerzas de poder que influyen en la dinámica de los elementos que lo conforman, la forma en que determinados grupos utilizan los recursos naturales básicos, los transforma, los alteran y generan, en el peor de los casos, la degradación progresiva de los mismos. (Hernández Garzón, 2019, pág. 96)

En este sentido, el análisis realizado por Hernández Garzón (2019) se centró en la explicación de las relaciones causales entre los procesos sociales (económicos, políticos, culturales) y sus efectos en el medioambiente, desde unaperspectivageopolítica (analizandolas relaciones de poder y los actores que participan en estas las interrelaciones que han incidido en la expresión de la problemática ambiental en el territorio) y ambiental (apropiación y uso de la naturaleza y sus recursos). Para tal efecto, construye una matriz donde se hacen evidentes las variables que se tienen en cuenta para el mencionado análisis que puede ser utilizada a la hora de plantear las líneas de acción de nuestra propuesta de Centro de Gestión Ambiental urbana en el barrio San Benito. 
TABLA 3. COMPONENTES PARA LA ELABORACIÓN DEL ANÁLISIS TERRITORIAL

\begin{tabular}{|c|c|c|}
\hline DIMENSIÓN & SECTOR & VARIABLES \\
\hline \multirow[t]{3}{*}{ NATURAL } & \multirow[t]{3}{*}{ Oferta ambiental del territorio } & Ecosistemas Estratégicos \\
\hline & & Oferta del Suelo \\
\hline & & Oferta del Recurso hídrico \\
\hline \multirow[t]{14}{*}{ SOCIAL } & \multirow[t]{3}{*}{ Política } & Tenencia de la tierra \\
\hline & & Asentamientos humanos \\
\hline & & Grupos de Poder \\
\hline & \multirow[t]{2}{*}{ Sistema económico } & Actividades económicas \\
\hline & & $\begin{array}{l}\text { Influencia de la economía } \\
\text { nacional y regional }\end{array}$ \\
\hline & \multirow[t]{2}{*}{ Cultura } & Valores e ideología \\
\hline & & Imaginarios urbanos y memoria \\
\hline & \multirow[t]{3}{*}{ Población } & Dinámica poblacional \\
\hline & & Migración \\
\hline & & $\begin{array}{l}\text { Distribución espacial de la } \\
\text { población }\end{array}$ \\
\hline & \multirow[t]{2}{*}{ Vivienda } & $\begin{array}{l}\text { Dinámicas en la construcción } \\
\text { de vivienda }\end{array}$ \\
\hline & & Programas de vivienda \\
\hline & \multirow[t]{2}{*}{ Crecimiento Urbano } & Expansión urbana \\
\hline & & $\begin{array}{l}\text { Procesos históricos de } \\
\text { ocupación del suelo }\end{array}$ \\
\hline & \multirow[t]{3}{*}{ Servicios Públicos } & Servicio de agua y alcantarillado \\
\hline & & Limpieza y saneamiento \\
\hline & & Gestión de residuos solidos \\
\hline
\end{tabular}

Fuente: Hernández Garzón (2019)

Molina Prieto, Suárez Serrano, \& Villa Camacho (2019) proponen un módelo multifactorial de bucle multidisciplinar para la sustentabilidad urbana, que reconoce los problemas ambientales generados por el crecimiento acelarado de la población urbana. En este sentido proponen ocho conceptos que fortalecen la sustentabilidad urbana a saber: ciclo cerrado, metabolismo urbano, economía circular, ecología industrial, red de valor, logística inversa, psicología ambiental y ecodiseño. Los autores concluyen que "para alcanzar la sustentabilidad urbana urge crear vasos comunicantes entre las disciplinas que inciden de manera directa en las dinámicas que dan vida a las ciudades contemporáneas."(pág. 76)
La energía, los materiales y el agua son transformados en las ciudades para generar bienes o servicios que permitan mantener el normal fun-cionamiento de la vida urbana. Pero, al transfor-marlos, se generan una serie de externalidades no deseadas: i) emisiones contaminantes genera-das por fuentes fijas y móviles; ii) residuos sólidos resultantes de proce-sos industriales, comerciales $y$ residenciales; iii) escombros generados por la industria de la construcción o por demolición de edificaciones obso-letas; y iv) residuos líquidos provenientes de la industria, la vivienda, el comercio y otras activi-dades, como las recreativas 
o las educativas. (Molina Prieto, Suárez

Serrano, \& Villa Camacho, 2019, pág. 79)

Según Molina Prieto, Suárez Serrano, \& Villa Camacho (2019) en lo concerniente a las emisiones de efecto invernadero, Bogotá originó el equivalente a 6,7 millones de toneladas de CO2 en 2014. En cuanto a residuos sólidos, Bogotá generó 2,3 millones de toneladas en 2014 y generó 223 millones de metros cúbicos de aguas residuales en 2014, y 12 millones de toneladas de escombros ese mismo año.

En este sentido, los conceptos que proponen Molina Prieto, Suárez Serrano, \& Villa Camacho (2019) desde un enfoque multidisciplinar buscan consolidar un modelo de gestión ambiental urbana. El primero de ellos procede de la ingenieria industrial y es el del ciclo cerrado; que se aplica a una variedad de actividades de recuperación: adquisición de productos usados, logística inversa, remanufactura, reparación y remarketing, entre otras. Se proponen cinco fases en la evolución del concepto y su aplicación en la ingeniería industrial: i) la era dorada de la remanufactura; ii) de la remanufactura a la valoración de la logística inversa, que contribuyó de manera fundamental a la solución de una serie de problemáticas económicas y ecológicas; iii) coordinación de la cadena de suministro inversa; iv) Cerrando el ciclo, perspectiva de diseño integrado que tiene implicaciones importantes en el mercado, pero exige el reconocimiento y la participación de un gran número de actuantes independientes que necesitan ser coordinados para lograr el potencial económico del sistema; y v) precios y mercados, fase actual que apenas está comenzando y su punta de lanza son los mercados verdes.

El segundo concepto propuesto por Molina Prieto, Suárez Serrano, \& Villa Camacho (2019) es el de "metabolismo urbano" construido por Abel Wolman, en 1965.
Wolman planteó dos cosas: i) que las ciudades cuentan con una serie de necesidades metabólicas para sustentar a sus habitan-tes, como alimentos, combustible, ropa, bienes duraderos, materiales de construcción, energía eléctrica, entre otras muchas; y ii) que el ciclo metabólico solamente se completa cuando los residuos y desechos originados por las actividades diarias son retirados y eliminados sin generar peligros $\mathrm{o}$ incomodidades a los habitantes de las ciudades. (pág. 81)

Wolman "centró su estudio en lo que denominó los tres problemas metabólicos más agudos: la provisión de un suministro adecuado de agua, la eliminación efectiva de las aguas residuales y el control de la contaminación del aire (Wolman, 1965)" citado por (Molina Prieto, Suárez Serrano, \& Villa Camacho, 2019, pág. 81)

El tercer concepto propuesto por Molina Prieto, Suárez Serrano, \& Villa Camacho (2019) es el de la economía circular entre sus principales alcances se encuentra:

i) permite transformar la función de los recursos en la economía; ii) los residuos industriales se convierten en insumos para otras industrias; iii) al concluir su ciclo de vida, los productos pueden ser reparados, reutilizados o mejorados, en lugar de desechados; iv) en un mundo caracterizado por los altos y volátiles precios de los recursos, ofrece enormes oportunidades de negocios; v) si se acelera su implementación a través de políticas públicas, puede contribuir a la mitigación del cambio climático, la escasez de agua y otros desafíos globales; vi) puede contribuir a aliviar las preocupaciones concernientes al acceso a recursos e insumos. (Molina Prieto, Suárez Serrano, \& Villa Camacho, 2019, págs. 81-82) 
El cuarto concepto propuesto por Molina Prieto, Suárez Serrano, \& Villa Camacho (2019) es el de Ecología Industrial

El concepto de ecología industrial se deriva de dos disciplinas: la ecología y la teoría de sistemas. Bajo los postulados de esas dos disciplinas busca estudiar el desarrollo y el comportamiento de los sistemas industriales desde el enfoque de los patrones de evolución de los sistemas naturales, lo que incluye: ciclo cerrado de materiales, principios evolutivos, resiliencia de los sistemas y retroalimentación dinámica (O’Rourke, Connelly y Koshland, 1996). Aunque son muchos los autores que han realizado aportes al concepto de ecología industrial, los objetivos que estableció John Ehrenfeld en 1994 continúan vigentes: i) mejorar las vías metabólicas para los procesos industriales y el uso de los materiales; ii) crear ecosistemas industriales de ciclo cerrado; iii) desmaterializar la producción industrial; iv) sistematizar los patrones de uso de energía; v) equilibrar los insumos y los productos industriales con la capacidad de los ecosistemas naturales; vi) alinear la política para ajustarse a la evolución del sistema industrial a largo plazo; vii) crear nuevas estructuras para acciones coordinadas, con vínculos comunicativos y de información (p. 16) citado por (Molina Prieto, Suárez Serrano, \& Villa Camacho, 2019, pág. 82).

El quinto concepto propuesto por Molina Prieto, Suárez Serrano, \& Villa Camacho (2019) es el de red de valor:

Coyle, Langley, Novak y Gibson (2013) definen la red de valor como una serie de empresas integradas que deben compartir información y coordinar la ejecución física para asegurar un flujo de bienes, servicios, información y dinero de forma efectiva. Otros autores, como Stock y Boyer (2009) vinculan una multiplicidad de integrantes y canales al concepto de red de valor. Destacan los flujos de recursos hacia adelante y hacia atrás -"aguas abajo" y "aguas arriba"- y señalan que es gracias al funcionamiento y la dinámica del sistema, que involucra a todos los participantes de la(s) red(es), como se logran suplir los requerimientos del cliente. (Molina Prieto, Suárez Serrano, \& Villa Camacho, 2019, pág. 82)

El sexto concepto propuesto por Molina Prieto, Suárez Serrano, \& Villa Camacho (2019) es el de logística inversa:

Para Cabeza (2012), la logística inversa: "abarca el conjunto de actividades logísticas de recogida, desmontaje y desmembramiento de productos ya usados o sus componentes, así como de materiales de distinto tipo y naturaleza con el objeto de maximizar el aprovechamiento de su valor, en sentido amplio de su uso sostenible $\mathrm{y}$, en último caso, su destrucción" (p. 26). Por último, Dyckhoff, Lackes y Reese (2013) consideran que la logística inversa comprende todas las actividades relacionadas con el manejo, el procesamiento, la reducción y la disposición de todos los residuos peligrosos y no peligrosos generados por la producción, además del embalaje $y$ el uso de los productos, incluido el proceso de distribución inversa, y destacan su función ecológica, puesto que, gracias a ella, se pueden evitar innumerables impactos negativos sobre el ambiente. (Molina Prieto, Suárez Serrano, \& Villa Camacho, 2019, pág. 83)

El séptimo concepto propuesto por Molina Prieto, Suárez Serrano, \& Villa Camacho (2019) es el de Psicología ambiental: 
No fue sino hasta la década de los noventa cuando se desarrolló una psicología ambiental vinculada a la conservación del ambiente. A partir de entonces, se invirtió la relación precedente para empezar a estudiar las relaciones conductaambiente, es decir, los efectos ambientales generados por las conductas humanas. De manera que existen dos enfoques bien diferenciados de la psicología ambiental: i) el que analiza los efectos del ambiente -natural o construido- sobre la conducta humana; y ii) el que estudia los efectos de la conducta humana sobre el ambiente físico y natural. (Molina Prieto, Suárez Serrano, \& Villa Camacho, 2019, pág. 83)

El ultimo concepto propuesto por Molina Prieto, Suárez Serrano, \& Villa Camacho (2019) es el de Ecodiseño:

Glavic y Lukman (2007) lo conciben como "un proceso de desarrollo de producto que tiene en cuenta su ciclo de vida completo, y considera aspectos ambientales en todas las etapas del proceso, buscando productos que generen el impacto ambiental más bajo posible a lo largo del ciclo de vida" (p. 1875). Mencionan, además, que el ecodiseño busca la reducción de materiales de entrada, la minimización de externalidades negativas a la salida, así como la reducción de los riesgos para la salud humana; y que se relaciona estrechamente con la evaluación del ciclo de vida, la ingeniería ambiental o verde, y los procesos de reutilización, reciclaje y remanufacturación. (Molina Prieto, Suárez Serrano, \& Villa Camacho, 2019, pág. 84)

En síntesis, todos los conceptos presentados por Molina Prieto, Suárez Serrano, \& Villa Camacho (2019) buscan los mismos objetivos; y hacen parte de procesos humanos, industriales y urbanos. Sin embargo, "Infortunadamente, esos conceptos teóricos, tan valiosos para el equilibrio ambiental, se han mantenido en distintas parcelas disciplinares, aislados, separados, encapsulados, encriptados, cerrados sobre sí mismos, o mejor: enclaustrados dentro de las murallas de sus propias disciplinas" (Molina Prieto, Suárez Serrano, \& Villa Camacho, 2019, pág. 85)

En consecuencia, urge que los conceptos que fortalecen la sustentabilidad urbana, aunque históricamente surgieron de distintas disciplinas, ahora se integren, se estructuren y se articulen, para que logren actuar como un sistema multidisciplinar unificado y compacto. Así, sus efectos serán más sólidos, eficaces y eficientes. (Molina Prieto, Suárez Serrano, \& Villa Camacho, 2019, pág. 85)

En este sentido, cobra vital importancia para nuestra propuesta de Centro de Gestión Ambiental desde un enfoque de sostenibilidad en el Barrio San Benito, el siguiente llamado

Las ciudades del siglo XXI están pasando por una época de grandes cambios. Uno de los más trascendentes y necesarios consiste en que los profesionales e investigadores de una disciplina, sin importar cuál sea, incursionen en otros campos de conocimiento, porque esa exploración les permite crear vasos comunicantes y redes interdisciplinares que: i) estimulan y fertilizan la creatividad humana (Florida, 2009); ii) facilitan la creación de nuevas formas de hacer las cosas -para hacerlas de manera sustentable-; iii) contribuyen con el desplome de los viejos paradigmas urbanos que no solo permanecen enquistados en el núcleo de la sociedad, sino en las percepciones y conductas de los ciudadanos. Paradigmas obsoletos e insustentables que impiden alcanzar un bienestar humano verdaderamente 
equitativo y ambientalmente sano y que, por tanto, urge desmantelar. Porque, mientras persistan, continuarán destruyendo la fuente de la energía, los materiales y el agua que tanto requieren la humanidad y las ciudades para sobrevivir, es decir, la naturaleza. (Molina Prieto, Suárez Serrano, \& Villa Camacho, 2019, pág. 87)

El libro Cambio climático, ciudad y gestión ambiental. Los ámbitos nacional e internacional. Es una obra colectiva editada por el Colegio de México, el propósito principal de este texto, además de reflexionar cerca de los problemas urbanos y cómo ellos comprometen la sustentabilidad. (Flórez Chávez, 2019, pág. 127).

Flórez Chávez (2019) realiza una reseña del mencionado libro elaborado desde el Centro de Estudios Urbanos y Ambientales (CEDUA) del Colegio de México (COLMEX), con la coordinación de José Luis Lezama (2018).

Menciona el coordinador de la obra Lezama (2018) que los diversos trabajos que integran la publicación citada consideran que el Desarrollo Sustentable es la respuesta a la racionalización económica imperante en la actualidad. Así mismo, la obra se ha dividido en tres apartados: 1. El cambio climático como asunto político, 2. Ciudad, Medio Ambiente y Gestión y 3. Medio ambiente: sociedad y política, para efectos de la presente investigación, se identifica la pertinencia de la sección 2 y algunos aspectos trabajados en la sección 3 .

El artículo Retos en el diseño de una política ambiental para la Zona Metropolitana del Valle de México: límites y oportunidades, concluye que, en los contextos metropolitanos mexicanos, la implementación de la política urbana se torna complicada, dado que no existen gobiernos urbanos o metropolitanos, ocasionando fragmentación social y político-administrativa y, una estructura sectorizada de la administración pública de los gobiernos locales y en general de los tres órdenes de gobierno lo que limitan la viabilidad y la eficacia en la implementación de políticas públicas (Graizbord y González Granillo, 2018), citado por (Flórez Chávez, 2019, pág. 125)

El texto Ciudades Sostenibles en México ¿qué hacer?, contextualiza el término Desarrollo Urbano Sostenible (DUS) en la agenda internacional y ofrece una propuesta metodológica a partir de la multidimensionalidad del desarrollo sustentable: económica, ambiental, política, poblacional, social, institucional y en la movilidad, inclusión y acceso a las oportunidades urbanas, con la finalidad de adoptar un plan sostenible en entornos que van desde lo metropolitano hasta las localidades de tamaño pequeño (Garrocho y Sobrino, 2018) citado por (Flórez Chávez, 2019, pág. 125). Sin embargo, como se señalo anteriormente, la visión del desarrollo sostenible, aunque importante es insuficiente en términos de los objetivos de la presente propuesta.

El trabajo La instrumentalización de la gestión integral de residuos sólidos urbanos en México: más allá de la regulación de los derechos, analiza el proceso de construcción de la acción pública de los residuos sólidos urbanos a partir de un modelo GIRSU, enmarcado en un sistema dinámico, donde los actores inmiscuidos en el tema buscan gestionar los desechos, que pase de basura inútil a un recurso valorable (Jiménez Martínez, 2018) citado por (Flórez Chávez, 2019, pág. 125). Este trabajo se constituye en un referente muy valioso a la hora de intentar gestionar los desechos generados mayoritariamente por la actividad económica en el Barrio San Benito.

El trabajo ¿Funciones y servicios ecosistémicos, ausentes en la política pública ambiental? Caso de estudio de la Ciudad de México, reconoce que la deforestación y degradación ambiental 
están presentes en la Ciudad de México y para contrarrestar dichas situaciones se han desarrollado diversos instrumentos de política pública ambiental (PPA). Lamentablemente muchos de estos programas se han aplicado sin basarse en principios ecológicos, por lo cual este tipo de políticas dificultan que se proteja a la naturaleza y se sigan considerando a los bosques solamente por su valor económico (Perevochtchikova y Rojo Negrete, 2018) citado por (Flórez Chávez, 2019, pág. 126). Se identifican pues, similitudes en la problemática mexicana con las enunciadas para el caso colombiano por Duquino-Rojas (2018), un referente para nuestra porpuesta de centro de Gestión Ambiental urbana con un enfoque de sustentabilidad en el barrio San Benito.

La universidad piloto de Colombia ha generado una producción investigativa importante a partir de su especialización en Gestión Ambiental Urbana, Valdés Osorio \& Salamanca Pedreros (2017), analizan los temas de resiliencia ambiental y movilidad urbana y su relación con el cambio climático. El enfoque que manejan es el de Gestión Ambiental Urbana encaminada a aportar en la construcción y formación de ciudades más resilientes, específicamente la ciudad de Bogotá D.C. se ha seleccionado como referente para analizar los fenómenos expuestos.

En los capítulos tres y cuatro, los mencionados autores plantean unos retos desde la gestión ambiental urbana para la adaptación al cambio climático y el mejoramiento de la movilidad urbana como apuesta para lograr ciudades sostenibles y resilientes en Colombia, identican que:

Uno de los mayores problemas en la ciudad, con respecto a los desafíos ambientales que enfrenta hoy en día, es la desinformación de la comunidad y de la realidad que se vive, en este sentido se propone empezar a ajustar procesos por medio de plataformas digitales a las cuales la comunidad tenga acceso sin necesidad de transportarse hasta las entidades públicas y privadas. La entidades públicas deben tener actualizadas todas sus plataformas de información; y reportar todos los días los datos necesarios con respecto al clima, los riesgos y los eventos naturales y antrópicos que puedan alterar la dinámica urbana. Esta información debe estar disponible a través de puntos de información digital ubicados en toda la ciudad que sean incluyentes y de fácil acceso. (Valdés Osorio \& Salamanca Pedreros, 2017, pág. 46).

\section{Así mismo, señalan:}

La idea de generar una simbología más moderna y accesible en la ciudad -que se adapte a la diversidad de condiciones de los ciudadanos por medio de un urbanismo incluyente con todas las personas que tienen una condición de discapacidad- fortalece la capacidad de resiliencia de estas personas en particular. Esto permite que los espacios públicos se conviertan en áreas de inclusión urbana y de interacción cultural. (Valdés Osorio \& Salamanca Pedreros, 2017, pág. 46)

\section{De otra parte:}

Con el fin de mejorar no solo la alimentación de las personas en la ciudad sino de su misma producción se debe incentivar la agricultura urbana desde la vivienda. Las granjas urbanas deben proyectarse en toda las comunidades, pero puede iniciar desde la casa. Sería más responsable con el ambiente producir el alimento dentro de la ciudad, ya que la huella ecológica que se genera por la importación de alimentos es un problema invisible a los ojos de las personas que residen en la ciudad, por esta razón es importante iniciar desde 
el hogar. Estos programas de agricultura urbana deben emprenderse también en lugares donde la demanda de alimentos es alta pero la oferta muy baja, por ejemplo, en las cárceles de todo el país los presidiarios demandan tres comidas al día como mínimo y es vasta la producción de basura; una gran ayuda, no solo para el Estado quien es el ente encargado de estos lugares, sino para la calidad ambiental de la ciudad, sería considerar la implementación de granjas dentro de las prisiones para que estas personas puedan producir su propio alimento sin necesidad de generar una carga en las urbes. (Valdés Osorio \& Salamanca Pedreros, 2017, págs. 46-47)

Un vacío evidenciado por Valdés Osorio \& Salamanca Pedreros (2017) en las ciudades es que no están preparadas para asumir los riesgos generados por las temporadas de lluvias y no cuentan con mecanismos de adaptación frente a estos fenómenos. "Para esto, se propone implementar la construcción de tanques de almacenamiento a nivel urbano durante la temporada de lluvias que puedan ser aprovechados en la temporada seca, en la que se ha empezado a vivir escasez de agua tanto adentro como afuera de la ciudad". (pág. 47).

En este sentido Valdés Osorio \& Salamanca Pedreros (2017) proponen también, construir parques inundables, que puedan mitigar el impacto de las inundaciones de manera rápida evitando situaciones de emergencia, y que a su vez los parques inundables puedan cumplir una función pública, de otra parte, proponen implementar "un sistema recolector de agua lluvia -que podrían implementarse en BogotáBasta con situar varios tanques, ya sea en zonas duras o zonas verdes, con el propósito de conducir el agua a su destino final y que ésta no se concentre generando inundaciones". (pág. 47-48)
Otro elemento importante que señalan Valdés Osorio \& Salamanca Pedreros (2017) es el relacionado con los procesos de construcción: "con responsabilidad, teniendo en cuenta cada una de las etapas desarrolladas dependiendo el nivel o escala de cada proyecto arquitectónico que se construya en la ciudad. En otras ciudades del mundo se han implementado nuevas tecnologías, nuevos productos y procedimientos más amigables ambientalmente hablando". (pág.49)

Otro aspecto importante que resaltan Valdés Osorio \& Salamanca Pedreros (2017), hace referencia a la recuperación de las zonas verdes y ecológicas que hay en la ciudad. Darles el tratamiento necesario, ya sea de: recuperación, conservación o preservación, en tanto se evidencie la afectación que estén sufriendo. Además de recuperar dichas zonas -que hacen parte del sistema de amortiguación natural en las ciudades (pág. 49)

Un elemento fundamental en términos de nuestra propuesta de Centro de Gestión ambiental con un enfoque de sostenibilidad es la propuesta realizada por Valdés Osorio \& Salamanca Pedreros (2017) de estructurar foros locales y la conformación de grupos técnicos, en cada barrio, en este caso el barrio San Benito que permitan optimizar el uso de las plataformas digitales, generar unurbanismo incluyente en el barrio, incentivar la agricultura urbana desde la vivienda, mitigar los riesgos generados por las temporadas de lluvias y el diseño de mecanismos de adaptación frente a estos fenómenos, consolidar proyectos de construcción sostenible, recuperación de las zonas verdes y ecológicas, donde puedan participar expertos en gestión ambiental urbana y se estructuren encuentros con los residentes donde se compartan experiencias y las situacionales actuales de los riesgos de vulnerabilidad.

Edelman \& Garrido Estévez (2019), realizan una investigación trata de alinear la teoría y la práctica 
contemporáneas de la gestión ambiental urbana para proponer soluciones a los problemas reales que enfrenta la ciudad de Lima Perú, analizan el contexto latinoamericano y trata de profundizar en distintas cuestiones relacionadas con temas como la reducción de la pobreza, la industria, las aguas residuales y el saneamiento, el agua, la energía, el transporte y las finanzas. Entre las propuestas que realizan se destacan, un proyecto de agricultura sostenible que permita ante la escasez de agua propia de la ciudad, que permite que los campos sean irrigados con aguas grises.

La FAO espera financiar este proyecto en coordinación con el sector industrial y con la

cooperación del sector deaguas residuales y saneamiento (The Many Benefits of Drip Irrigation, 2017). Los campos serían irrigados con aguas grises del sector de aguas residuales, mientras que el sector industrial proveería del equipo necesario al igual que de la capacitación que asegure el mejor rendimiento para los cultivos. Se dedicaría un año al estudio y diseño, y se implementaría en los cuatro años restantes. Los US $\$ 4.2$ millones de dólares se usarían para construir la infraestructura del sistema de irrigación y vendrían de préstamos de bancos comerciales locales y con garantía de la FAO. El costo total de este proyecto sería de US $\$ 160,000$ y $\mathbf{S} / 129,827$, que cubriría el estudio y diseño que se haría durante el primer año, mientras que el resto de los US\$4 millones, serían utilizados para la construcción. (Edelman \& Garrido Estévez, 2019, pág. 84)

Lo anterior nos permite identificar posibles financiadores de los proyectos de agricultura sustentable que pretendemos estructurar en el barrio San Benito. Un segundo proyecto que proponen Edelman \& Garrido Estévez (2019) es "construir una planta de reciclaje que reduzca la contaminación y promueva un ambiente más saludable. Se tardaría un año en hacer un estudio, otro año en la construcción y tres en implementar la planta." (pág.85)

Otro proyecto que proponen Edelman \& Garrido Estévez (2019) para reducir la ineficiencia en el uso del agua por parte de agricultores y otras familias en Lima, es la implementación de un programa de educación sobre el agua para la comunidad. Este programa tiene dos partes; una, dirigida a los pequeños agricultores al margen del área metropolitana de Lima, y otra, dirigida a niños de 10 y 11 años.

\section{INDICADORES PARA EVALUAR LA GESTIÓN AMBIENTAL URBANA.}

Para finalizar esta revisión sistemática de literatura, orientada hacia los elementos constitutivos de una propuesta de Centro de Gestión Ambiental desde un enfoque de sustentabilidad, es necesario hacer referencia a la construcción de indicadores, que permitan evaluar la gestión del mencionado centro.

Como señalan Karis, Mujica, \& Ferraro (2019) las ciudades enfrentan desafíos de sustentabilidad que hacen necesario generar información ambiental a través del uso de indicadores, los autores estructuran una propuesta de indicadores que den cuenta de los Servicios Ecosistémicos Urbanos culturales, "haciendo especial hincapié en aquellos que se obtienen de la interacción directa con la naturaleza (entre los que se destacan las oportunidades para la realización de actividades deportivas y de ocio, la contemplación de la naturaleza y la educación y la investigación)".(pág.11).

Para generar información sobre los SEU culturales, podrían cuantificarse los espacios verdes públicos, de gran importancia por su accesibilidad, y también otros tipos de áreas verdes, como 
las situadas en áreas residenciales, que en algunos casos han demostrado ser las principales proveedoras de algunos servicios por su mayor superficie (Karis, Mujica, \& Ferraro, 2019, pág. 13)

Karis, Mujica, \& Ferraro (2019) diseñan cuatro indicadores para dar cuenta de los servicios ecosistemicos urbanos en la zona urbana y periurbana de la ciudad de Mar del Plata Argentina:

Tabla 4 Conjunto de indicadores ambientales SEU Mar del Plata

\begin{tabular}{|c|c|c|}
\hline 1 & $\begin{array}{c}\text { INDICADOR } \\
\text { Superficie verde pública por habitante }\end{array}$ & $\begin{array}{l}\text { DESCRIPCIÓN } \\
\text { El indicador mide la extensión de las áreas verdes } \\
\text { públicas existentes y la relación con el número de } \\
\text { habitantes. } \mathbf{m} 2 / \text { hab. }\end{array}$ \\
\hline 2 & $\begin{array}{l}\text { Distribución de la superficie } \\
\text { verde pública. }\end{array}$ & $\begin{array}{l}\text { El indicador mide la participación de cada zona en la } \\
\text { superficie total de áreas verdes públicas del área de } \\
\text { estudio. } \\
\text { - Plazas barriales: espacios abiertos de superficie de } \\
\text { entre } 1000 \text { m2 y } 3,5 \text { ha donde predominan la cobertura } \\
\text { vegetal y los elementos naturales, de acceso libre, } \\
\text { cuyas funciones principales son la recreación, el } \\
\text { contacto con la naturaleza y la interacción social. } \\
\text { - Plazas y parques urbanos: espacios abiertos de } \\
\text { superficie de entre } 3,5 \text { y } 10 \text { ha donde predominan la } \\
\text { cobertura vegetal y los elementos naturales, de acceso } \\
\text { libre, cuyas funciones principales son la recreación, el } \\
\text { contacto con la naturaleza y la interacción social. } \\
\text { - Parques grandes: espacios abiertos de superficie } \\
\text { mayor a } 10 \text { ha donde predominan la cobertura vegetal } \\
\text { y los elementos naturales, de acceso libre, cuyas } \\
\text { funciones principales son la recreación, el contacto } \\
\text { con la naturaleza y la interacción social. } \\
\text { - Áreas de reserva natural: espacios naturales que por } \\
\text { su fragilidad, importancia o singularidad son objeto de } \\
\text { protección legal para garantizar su conservación. Se } \\
\text { consideran para el cálculo de los indicadores aquellos } \\
\text { de propiedad pública, excluyéndose los barrios } \\
\text { residenciales declarados reserva forestal. }\end{array}$ \\
\hline
\end{tabular}




\begin{tabular}{|c|c|c|}
\hline 3 & Superficie no impermeabilizada & $\begin{array}{l}\text { El indicador mide el porcentaje de superficie de suelo } \\
\text { no impermeabilizado. }\end{array}$ \\
\hline 4 & $\begin{array}{l}\text { Índice de vegetación diferencial } \\
\text { normalizada (NDVI) }\end{array}$ & $\begin{array}{l}\text { Se utiliza el NDVI para evaluar la superficie de } \\
\text { suelo cubierta de vegetación en espacios públicos y } \\
\text { privados. } \\
\qquad N V D I=I R C-R / \text { IRC +R } \\
\text { Donde IRC es la reflectividad en el infrarrojo cercano } \\
\text { (banda } 5 \text { del Landsat } 8 \text { ) y R es la reflectividad en el } \\
\text { rojo (banda } 4 \text { del Landsat } 8 \text { ). } \\
\text { El NDVI adopta valores entre -1 a } 1 \text { y puede ser } \\
\text { interpretado de la siguiente forma: } \\
\text { - Valores entre -1 y } 0 \text { corresponden a agua y nubes. } \\
\text { - Valores cercanos a cero corresponden principalmente } \\
\text { a afloramientos rocosos, construcciones y terrenos } \\
\text { desnudos o sin vegetación. } \\
\text { - Valores moderados (aproximadamente de } 0,4 \text { a } 0,7 \text { ) } \\
\text { representan terrenos con arbustos y } \\
\text { prados. } \\
\text { - Valores altos (> a } 0,7 \text { ) indican vegetación frondosa } \\
\text { y en buen estado, asociada principalmente a } \\
\text { forestaciones. }\end{array}$ \\
\hline
\end{tabular}

Fuente: (Karis, Mujica, \& Ferraro, 2019)

Otra aproximación a los indicadores se encuentra en el estudio de Moreno Sánchez (2014), para quien un indicador de sustentabilidad

Va más allá de un recuento o medición estadística, trata de identificar y medir el impacto humano sobre el ambiente y sus componentes a partir de una referencia; permite inferir la capacidad del ambiente para ciertas actividades que tienen que ver con un tipo de desarrollo o crecimiento económico durante un largo tiempo. (pág.164).

\section{Así mismo:}

Se han generado múltiples datos relativos a la capacidad de carga de los ecosistemas, así como de la viabilidad económica de las actividades productivas que permitan a quien las realiza satisfacer sus necesidades primordiales. En la mayor parte de los casos, las evaluaciones refieren un estado de pobre sustentabilidad ambiental, lo cual pone en riesgo el futuro de la especie humana. Los indicadores de sustentabilidad se han convertido en una importante herramienta de toma de decisiones para gobiernos nacionales y locales, comunidades y 
actores sociales involucrados (Hammond et al., 1995). En cuanto a la "dimensión social" de la sustentabilidad, como lo han definido numerosos autores (Azar et al., 1996), son pocas las evaluaciones que van más allá de indicadores de la satisfacción de necesidades básicas, aunque existen métodos cuantitativos para estimar, por ejemplo; la distribución del ingreso, o bien, índices compuestos (índice de desarrollo humano) que reflejan el acceso de la población a servicios básicos como educación, salud e ingresos per cápita (UNDP, 2004). Citados por (Moreno Sánchez, 2014, pág. 164)

En cuanto al sustento teórico de los indicadores Moreno Sánchez (2014) señala

En el ámbito de la sustentabilidad urbana toma relevancia generar y conocer indicadores que permitan conocer la condición urbana, demográfica, económica y social a nivel local para que la sociedad civil, y los tomadores de decisiones puedan traducirse en acciones de estado que permitan la integración de variables socioeconómicas y físico-naturales en el análisis ambiental local y regional. El análisis y medición de la sustentabilidad a través de indicadores ha estado realizándose por los países mediante un marco ordenador estructurado en tres categorías de información: Presión-Estado-Respuesta (PER), diseñado y dado a conocer por la Organización para la Cooperación y el Desarrollo Económico (OCDE) en el año de 1993 (López, 2009: 38). (pág. 176)

Moreno Sánchez (2014) reconoce la existencia de una fuerte discusión acerca de los indicadores generados para analizar y estudiar la sustentabilidad en territorios urbanos. En nuestra propuesta de Centro de Gestión ambiental urbana desde un enfoque de sustentabilidad en el Barrio San Benito, en consonancia con este autor, sustentamos los indicadores en el nivel urbano en una localidad, pero somos conscientes que su efecto e impacto rebasa las barreras tradicionales administrativas-jurídicas de un territorio $y$, en todo caso, su mejor expresión se puede evaluar en la expresión de la calidad de vida de una sociedad a nivel local.

El estudio realizado por Moreno Sánchez (2014) en Chimalhuacán construyó los siguientes indicadores

Tabla 5. Indicadores generados en Chimalhuacan

\begin{tabular}{|c|c|c|}
\hline Indicador Económico & Indicador Ambiental & Indicador social \\
\hline $\begin{array}{l}\text { Población Economicamente } \\
\text { activa en el municipio PEA }\end{array}$ & $\begin{array}{l}\text { Porcentaje de Erosión del suelo } \\
\text { en la última década }\end{array}$ & Índice general de pobreza \\
\hline $\begin{array}{l}\text { Salario mínino promedio en el } \\
\text { municipio }\end{array}$ & $\begin{array}{l}\text { Superficie de área o áreas } \\
\text { protegidas }\end{array}$ & $\begin{array}{l}\text { Demanda de la fuerza de } \\
\text { trabajo }\end{array}$ \\
\hline $\begin{array}{l}\text { Gasto promedio de familia en } \\
\text { energía (luz) }\end{array}$ & $\begin{array}{l}\text { Superficie de cambio de uso de } \\
\text { suelo en un periodo determinado } \\
\text { de } 10 \text { a } 20 \text { años }\end{array}$ & $\begin{array}{l}\text { Vulnerabilidad en servicios } \\
\text { en vivienda (agua potable y } \\
\text { drenaje) }\end{array}$ \\
\hline $\begin{array}{l}\text { Número de bancos en el } \\
\text { municipio }\end{array}$ & $\begin{array}{l}\text { Plantas de tratamiento de agua } \\
\text { potable registradas y en servicio }\end{array}$ & Densidad de población \\
\hline PEA por sector económico & $\begin{array}{l}\text { Estudios y disponibilidad de agua } \\
\text { en el subsuelo }\end{array}$ & $\begin{array}{l}\text { Tasa promedio de escolaridad } \\
\text { de la población muncipal }\end{array}$ \\
\hline
\end{tabular}




\begin{tabular}{|l|l|l|l|}
\hline $\begin{array}{l}\text { Número de vehículos } \\
\text { automotores registrados }\end{array}$ & $\begin{array}{l}\text { Superficie dedicada a la } \\
\text { protección, conservación y yón del gobierno local } \\
\text { recarga de mantos acuíferos. }\end{array}$ & $\begin{array}{l}\text { en materia de desarrollo } \\
\text { sustentable }\end{array}$ \\
\hline
\end{tabular}

Fuente: (Moreno Sánchez, 2014)

\section{CONCLUSIONES E IMPLICACIONES DE POLÍTICA}

El trabajo de campo, realizado desde el Semillero de Investigación Contabilidad y Consecuencias Ambientales de la Cultura del Programa de Contaduría UVD de la Corporación Universitaria Minuto de Dios, es el que nos anima proponer la creación de un Centro de Gestión ambiental desde un enfoque de sustentabilidad en el Barrio San Benito.

La propuesta de Centro de Gestión ambiental urbano desde un enfoque de sustentabilidad en el barrio San Benito, implica acciones de conservación, defensa, protección y mejora del medio ambiente, a partir de un enfoque interdisciplinario y global. se refiere a la gestión de los recursos naturales renovables y los problemas ambientales que se presentan en el barrio y sus efectos en Bogotá-Región o regiones vecinas.

La propuesta busca articularse con el Estado en su dimensión local, regional y nacional y los actores sociales, para desde un enfoque de sustentabilidad apoyar la gestión territorial, las políticas ambientales y las políticas o planes sectoriales que tienen relación o afectan el medio ambiente en este ámbito urbano con el objeto de lograr la protección y funcionamiento de los ecosistemas y el mejoramiento de la calidad de vida de la población, dentro de un marco de ciudad sostenible.

Reconociendo que es un avance el diseño de una política de gestión ambiental urbana, es necesario señalar que dicha política se ha realizado desde un enfoque de sostenibilidad débil (desarrollo sostenible) que busca enfrentar la crisis ambiental desde las coordenadas de la globalización neoliberal.

$\mathrm{Si}$ bien las áreas urbanas son vistas como expresión de oportunidades de desarrollo económico y social, también es cierto que la conformación del sistema urbano colombiano se ha dado con una escasa planificación ambiental o de consideraciones ambientales, lo que ha derivado en innegables costos para el medio ambiente, tanto por los desordenados procesos de ocupación, como por las fuertes demandas de recursos que conllevan.

En acuerdo con Duquino-Rojas (2018) se identifica que la postura adoptada por la institucionalidad, evidencia una subyugación irrestricta a la retórica del desarrollo sostenible impuesta desde los espacios de poder transnacional, que hace que Colombia y Bogotá lleven 25 años sujetas a una política pública que, a la luz de los acontecimientos, ha fracasado, y no ha logrado mediar las disputas territoriales y económicas entre los intereses privados y públicos por los recursos naturales, y los valores ambientales (pág. 143). En este sentido se constituye en un referente en términos del enfoque y las variables a tener en cuenta por nuestra propuesta.

Una nueva política pública ambiental debe estar basada en el derecho inalienable a la ciudad para todos los integrantes de la sociedad y en la reivindicación de unos valores humanos que trasciendan las intenciones políticas, la normatividad y la planeación urbana para convertirse en herramientas reales de empoderamiento social para la gestión y la administración de la ciudad. (Duquino-Rojas, 2018, pág.149). Esto es precisamente lo que buscamos con la creación del Centro de Gestión 
urbana desde un enfoque de sustentabilidad en el Barrio san Benito.

En consonancia con el análisis realizado por Hernández Garzón (2019) nuestra propuesta de Centro de Gestión Ambiental desde un enfoque de sustentabilidad tendrá en cuenta las dimensiones sectores y variables propuestas por su investigación.

Siguiendo a Molina Prieto, Suárez Serrano, \& Villa Camacho (2019) comprendemos las necesidad de un abordaje multidisciplinar para la sustentabilidad urbana, nuestra propuesta busca aplicar de manera integrada los ocho conceptos que fortalecen la sustentabilidad urbana a saber: ciclo cerrado, metabolismo urbano, economía circular, ecología industrial, red de valor, logística inversa, psicología ambiental y ecodiseño.

La gestión ambiental urbana a la que pretendemos contribuir toma como propios los retos propuestos por Valdés Osorio \& Salamanca Pedreros (2017) desde la gestión ambiental urbana para la adaptación al cambio climático y el mejoramiento de la movilidad urbana como apuesta para lograr ciudades sostenibles y resilientes en Colombia, a saber consolidar plataformas digitales para el flujo de información ambiental hacia la comunidad, generar una simbología más moderna y accesible en el barrio San Benito, incentivar la agricultura urbana, implementar la construcción de tanques de almacenamiento durante la temporada de lluvias que puedan ser aprovechados en la temporada seca, construir parques inundables, que puedan mitigar el impacto de las inundaciones de manera rápida evitando situaciones de emergencia, controlar los procesos de construcción: "con responsabilidad, recuperación de las zonas verdes y ecológicas que hay en el barrio. Así mismo darles el tratamiento necesario, ya sea de: recuperación, conservación o preservación, en tanto se evidencie la afectación que estén sufriendo, estructurar foros conformar grupos técnicos, en el barrio.

En lo referente a la construcción de indicadores, que permitan evaluar la gestión de nuestro centro de gestión ambiental urbana desde un enfoque de sustentabilidad, implementaremos en primera instancia los cuatro indicadores propuestos por Karis, Mujica, \& Ferraro (2019) a saber Superficie verde pública por habitante, Distribución de la superficie verde pública, superficie no impermeabilizada y el Índice de vegetación diferencial normalizada (NDVI).

En paralelo siguiendo a Moreno Sánchez (2014), se implementarn para evaluar la sustentabilidad urbana del Barrio San Benito los siguientes indicadores: Porcentaje de Erosión del suelo en la última década, Superficie de área o áreas protegidas, Superficie de cambio de uso de suelo en un periodo determinado de 10 a 20 años, Plantas de tratamiento de agua potable registradas y en servicio, Estudios y disponibilidad de agua en el subsuelo, Superficie dedicada a la protección, conservación y recarga de mantos acuíferos.

\section{REFERENCIAS BIBLIOGRÁFICAS}

Ariza Buenaventura, D. (2007). Luces y sombras en el "poder constitutivo de la contabilidad ambiental". Revista Facultad de Ciencias Económicas: Investigación y Reflexión, XV(2), 45-60.

Ariza Ruiz, E. D. (2019). CONTABILIDAD Y CONSECUENCIAS AMBIENTALES DE LA CULTURA. En V. Autores, Libro de investigación: Apropiación, gestión y uso edificador del conocimiento (págs. 247-259). Santiago de Cali, Valle del Cauca, Colombia- New York, EE UU.: Editorial REDIPE (95857440), New York - Cali Coedición: Universidad de San Buenaventura. 
Bonet, E. (2 de 11 de 2016). Cierre de curtiembres en San Benito. El Espectador. Obtenido de http://www.elespectador.com

Carter, T. R. (1996). Adapting to climate changue: an international perspective. Nueva York: Springuer - Velarg.

Cuberos, E. R.-S. (2009). Niveles de cromo $y$ alteraciones de salud en una población expuesta a las actividades de curtiembres en Bogotá, Colombia. Revista de Salud Pública, 11(2), 278289.

Duquino-Rojas. (2018). Sustentabilidad ambiental urbana, alternativas para una política pública ambiental. Bitácora Urbano Territorial, 1(28), 141-149.

Edelman, D. J., \& Garrido Estévez, P. (2019). La Gestión Ambiental Urbana De Lima, Perú. European Scientific Journal, 15(5), 78-118. doi:10.19044/esj.2019. v15n5p78

Flórez Chávez, R. (2019). Cambio climático, ciudad y gestión ambiental. Los ámbitos nacional e internacional. Economía y Sociedad(39), 123-127.

Garcia, M. L., \& Vergara, J. M. (2000). La evolución del concepto de sostenibilidad y su introducción en la enseñanza de las ciencias. Revista de investigación y experiencias didácticas, 18(3), 473-486.

Gorz, A. (2012). Ecológica. Buenos Aires: Capital Intelectual.

Hernández Garzón, M. (2019). Análisis territorial de la problemática ambiental urbana: el caso del municipio de Facatativá,Cundinamarca, Colombia (1980-2010). Perspectiva Geográfica, 24(1), 92-115. doi:10.19053/01233769.9074
Hernandez Vargas, I., \& Moreno Sierra, M. A. (2017). APOYO EN LA FORMULACIÓN E IMPLEMENTACIÓN DEL PROYECTO AMBIENTAL INTERINSTITUCIONAL DIRIGIDO POR LA SUBDIRECCIÓN LOCAL DE TUNJUELITO DE LA CIUDAD DE BOGOTÁ D, C. Bogotá, Bogotá D.C, Colombia: Universidad Distrital Francisco José de Caldas.

Karis, C. M., Mujica, C. M., \& Ferraro, R. (2019). Indicadores ambientales y gestión urbana. Relaciones entre servicios ecosistémicos urbanos y sustentabilidad. Cuaderno Urbano. Espacio, Cultura, Sociedad, 27(27), 9-30.

Leff, E. (2009). Universidad, saber ambiental y sustentabilidad. Bogotá: Editorial Pontificia Universidad Javeriana.

Lezama, J. L. (2018). Cambio climático, ciudad y gestión ambiental.: Los ámbitos nacional $e$ internacional. Ciudad de México: El Colegio de Mexico AC. Centro de Estudios Demográficos, Urbanos y Ambientales.

Ministerio de Ambiente, Vivienda y Desarrollo Territorial. (2018). Política de Gestión ambiental Urbana. Bogotá, D.C. Bogotá.

Molina Prieto, L. F., Suárez Serrano, M., \& Villa Camacho, M. E. (2019). Bucle multidisciplinar para la sustentabilidad urbana. Revista de Arquitectura, 2(2), 76-88. Obtenido de https:// revistadearquitectura.ucatolica.edu.co/ article/view/2048

Montes Medina, P. A. (2018). Diseño de un modelo de Balance Scorecard ambiental para una curtiembre en el barrio de San Benito. Bogotá, Cundinamarca, Colombia: Corporación Universitaria Minuto de Dios. Obtenido de https:// 
repository.uniminuto.edu/bitstream/ handle/10656/7347/UVD-TCP_ MontesMedinaPaulaAndrea_2018. pdf?sequence $=1$

Moreno Sánchez, E. (2014). Indicadores para el estudio de la sustentabilidad urbana en Chimalhuacán, Estado de México. Estudios Sociales, XXII(43), 160-183.

Narciso, C. F. (2018). Estructura Ecológica Urbana: planeamiento y gestión urbana en la Ciudad de México. Estoa, 7(12), 137-147. doi: 10.18537/est.v007.n012. a12

Ramírez, J. A. (2014). Evaluación de la gestión integral de residuos sólidos en las curtiembres de San Benito. Universidad Militar Nueva Granada.

Rivera, M. A. (2007). La responsabilidad social corporativacomo resultante de uncambio en los imaginarios del contador público: del mecanicismo a la complejidad de las organizaciones. Contaduría Universidad de Antioquia(51), 65-87.

Romero Manrique, M., \& Leguizamón Cruz, L. J. (2016). INNOVACIÓN $Y$ COMPETITIVIDAD EN LA AGLOMERACIÓN DE CURTIEMBRES DEL BARRIO SAN BENITO EN BOGOTÁ. Bogotá, Bogotá D.C, Colombia: Universidad de La Salle.

Valdés Osorio, G., \& Salamanca Pedreros, A. (Febrero de 2017). Ciudades sostenibles, seguras y resilientes: Retos para la Gestión Ambiental Urbana en cambio climático y movilidad. Bogotá, Bogotá, D.C, Colombia: Universidad Piloto de Colombia.

Valenzuela Jimenez, L. F. (Enero-Junio 2017). Reflexiones sobre los conceptos Desarrollo Sustentable y Desarrollo
Sostenible. Relaciones con la Responsabilidad Social Organizacional. (RSO). Teuken Bidikay, 8(10), 211-229.

Vásquez Paniagua, J. A. (2009). Metodología para implementar un modelo de responsabilidad social empresarial (RSE) en la industria de la curtiembre en Colombia. Contabilidad y Negocios.

Zorro Sánchez, O. A. (2020). Posición contable de empresas de San Benito, frente al daño ambiental. En J. G. Zecua, K. Flores Tuxpan, \& G. R. Armas, Investigaciones para el fortalecimiento y competitividad de las empresas (págs. 106 -123). Tlaxcala: Universidad Autónoma de Tlaxcala. 